\title{
Ehrenmitglied der europäischen Histaminforscher
}

\section{Die European Histamine Research Society hat anläßlich ihrer 32. Jahrestagung im niederländischen Noordwijk Leeuwen- horst Prof. Dr. med. Wolfgang Schmutzler, Aachen, die Ehren- mitgliedschaft verliehen.}

D ie European Histamine Research Society (EHRS) wurde 1972 in Paris gegründet und ist die erste wissenschaftliche Gesellschaft Europas, die als solche in Brüssel anerkannt und nach belgischem Recht als gemeinnützig eingetragen ist. Die EHRS führt Wissenschaftler verschiedener Disziplinen zusammen, die an den Wirkungen und der Bedeutung von Histamin sowie verwandten Forschungsthemen interessiert sind. Sie fördert auf diese Weise die interdisziplinäre Kooperation von pharmazeu-

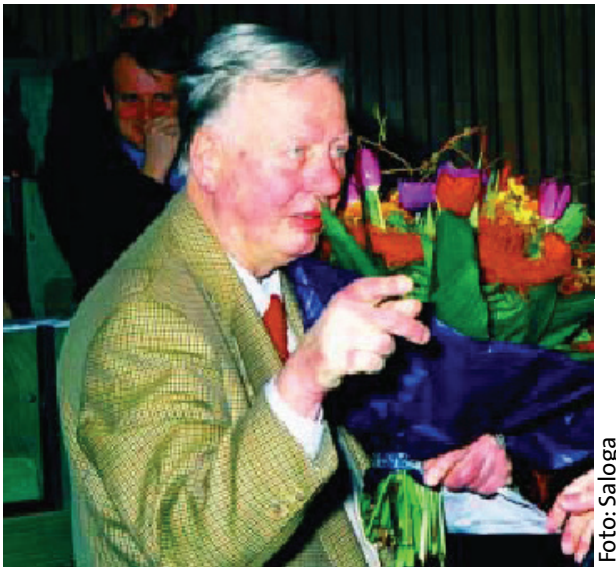

Prof. Dr. Wolfgang Schmutzler anlässlich einer Ehrung zu seinem 70. Geburtstag im März 2003 tischen Chemikern, Biochemikern, Biologen, Pharmakologen, Physiologen, Neurophysiologen, Immunologen sowie Klinikern aus Aller- gologie, Innerer Medizin, Anästhesiologie und Chirurgie. Die Gesellschaft führte bereits zu Zeiten des „Eisernen

\section{Internationale Neurodermitisstudie}

Die Klinik für Dermatologie und Allergologie am Biederstein, gehörend zum Klinikum rechts der Isar, München, ist an einer klinischen Studie zur Verhütung des Asthmas bei Kindern mit atopischem Ekzem beteiligt. Ausgangspunkt für die Studie ist die Tatsache, dass vor allem bei Kleinkindern mit Neuro-

dermitis das Risiko, später an Asthma zu erkranken, besonders hoch ist. So liegt es bei Kindern mit Ekzemen drei- bis fünfmal höher als bei anderen Kindern. Das eingesetzte Medikament ist sehr gut verträglich und seit längerer Zeit in Deutschland zugelassen.

Die Studie wird weltweit durchgeführt, insgesamt sind elf Länder einbezogen, neun davon in Europa. 500 Kinder sollen insgesamt in die Studie aufgenommen werden. Die gesuchten Kinder sollen bei Teilnahmebeginn im Alter zwischen 12 und 24 Monaten sein, insgesamt werden sie dann über ca. 18 Monate behandelt. Mindestens ein Familienmitglied muss an Asthma, Heuschnupfen oder Neurodermitis leiden oder in der Kindheit daran gelitten haben. Im Jahr 2006 soll die Studie abgeschlossen sein.

Klinik und Poliklinik für Dermatologie und Allergologie am Biederstein der TU München Dr. Mirjam Vogel/Dr. Stephan Fischer, Biedersteiner Straße 29, 80802 München Tel.: (o 89) 41 40-33 26

Tel. mobil: (01 75) 4537699 gocmes mSTAMNOLOCOAUM EMEORAROAUM

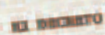

cocoinuen Genven ans

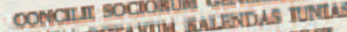

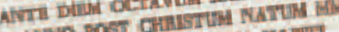

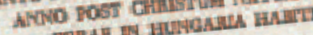

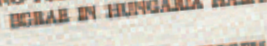
phorssonen dochonm VOLGGANGUM SCHMUTZLR

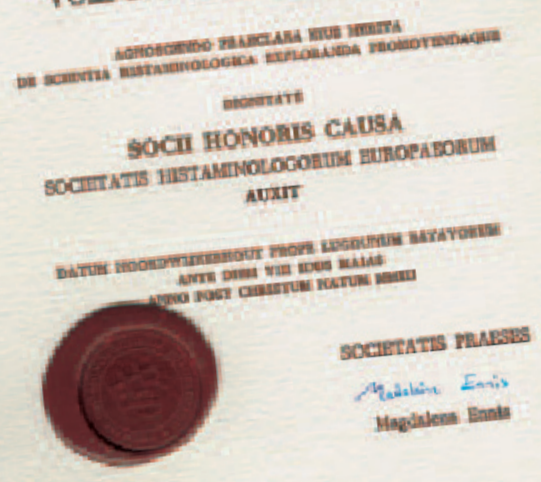

Die in Latein verfasste Urkunde zur Ehrenmitgliedschaft - Lugdunum Batavorum ist das heutige Leiden in den Niederlanden.

Vorhangs" stets Wissenschaftler aus Ost und West zusammen. An der vergangenen Jahrestagung 2003 nahmen 170 Wissenschaftler aus insgesamt 27 Ländern teil. Die Jahrestagung 2004 wird in Deutschland stattfinden - weitere Informationen unter www.ehrs.org.uk im Internet.

Prof. Schmutzler erhielt die Ehrenmitgliedschaft aufgrund seiner Forschungen auf dem Gebiet des Histamins. Er war von 1987 bis 1990 Präsident der DGAI, seit 1993 ist er Mitglied des Herausgeber-Kollegiums des Allergo Journal.

Bemerkenswert ist die Urkunde zur Ehrenmitgliedschaft in Latein, vor allem da Englisch die Verhandlungsund Umgangssprache der Gesellschaft ist. Dies soll wohl dokumentieren, dass man nicht die Absicht hat, der Gesellschaft angelsächsisch-amerikanisches Reglement aufzuzwingen. Die Satzung der EHRS wiederum ist übrigens in Französisch abgefasst. 